\title{
Mass Correlated Acceleration In a Reflectron MALDI TOF Mass Spectrometer: An Approach for Enhanced Resolution Over a Broad Mass Range
}

\author{
Slava V. Kovtoun, ${ }^{*}$ Robert D. English, and Robert J. Cotter \\ Middle Atlantic Mass Spectrometry Laboratory, Department of Pharmacology and Molecular Sciences, \\ The Johns Hopkins University School of Medicine, Baltimore, Maryland, USA
}

\begin{abstract}
Compared to continuous extraction, pulsed extraction (PE) of ions formed by matrix-assisted laser desorption/ionization (MALDI) in time-of-flight (TOF) mass spectrometers significantly improves mass resolution. Parameters such as extraction voltage, delay time, and correction pulse must be varied, however, to achieve optimum mass resolution over a broad mass range because the PE method is mass dependent. We previously reported a novel method, mass correlated acceleration (MCA), which we have now combined with a reflectron MALDI TOF mass spectrometer to further enhance mass resolution over a broader mass range. Unlike the PE method, MCA is not mass dependent and high resolution mass spectra can be achieved with a single tuning of instrument parameters. The ions may be brought into focus simultaneously, i.e., the multi-channel recording advantage can be more fully realized. The MCA dual-stage ion source design includes an extraction pulse region and an acceleration region that contains a time-dependent waveform correlated with mass. We demonstrate the validity of this novel technique with applications in peptide mixture analysis and protein digests of lysozyme and bovine serum albumin. (J Am Soc Mass Spectrom 2002, 13, 135-143) (c) 2002 American Society for Mass Spectrometry
\end{abstract}

$\mathrm{M}$ atrix-assisted laser desorption/ionization (MALDI) [1,2] is effective in the ionization of large biomolecules (peptides, proteins, carbohydrates, and oligonucleotides) and other polymers in mass spectrometry. The development of MALDI sources has sparked renewed interest in the time-offlight (TOF) mass analyzer, an excellent counterpart to pulsed laser sources in MALDI. In addition, TOF analyzers offer the multichannel recording advantage capable of recording the entire mass range simultaneously (i.e., no scanning of the mass analyzer is required).

Historically, the TOF analyzer has had the reputation of providing low mass resolution and, subsequently, poor mass accuracy. Uncertainties in time of ion formation, initial position, and kinetic energy contribute to the actual flight time. Hence, peak broadening (mass resolution) is affected by these conditions. To compensate for the initial temporal, spatial, and velocity ion distributions, Wiley and McLaren [3] introduced timelag focusing, a dual-stage ion source which provided

Published online November 30, 2001

Address reprint requests to Dr. R. J. Cotter, Mid-Atlantic Mass Spectrometry Laboratory, Department of Pharmacology and Molecular Sciences, The Johns Hopkins University School of Medicine, 725 North Wolfe Street, Baltimore, MD 21205, USA. E-mail: rcotter@jhmi.edu

*Present address: ThermoFinnigan, 355 River Oaks Pkwy., San Jose, CA 95134. first-order space focusing (with the detector positioned at an optimum distance) and permitted higher mass resolution measurements. Similar to what we now know as pulsed extraction (PE) or delayed extraction (DE), time-lag focusing used a time delay between the ionization pulse and the extraction pulse. Its more recent counterpart greatly improves the quality of MALDI mass spectra [4-8]. A disadvantage to PE focusing, however, is its mass dependency: each mass is focused at a different time delay and/or extraction pulse amplitude. Therefore, one or both of these parameters need to be varied in order to obtain mass spectra containing peaks with optimum resolution across the entire mass range. There have been considerable improvements in mass accuracy and mass resolution over a broad mass range [9-14]; however, our approach differs in that it utilizes a simple extraction pulse in the first region of a dual extraction source to focus a reference mass, and a separate waveform in the second (acceleration) region to focus all ions with masses lower than the reference mass.

\section{Theory}

The theory of MCA has been explained previously [15-17]. The concept of the method was described by Kovtoun employing both single-stage [15] and dual- 


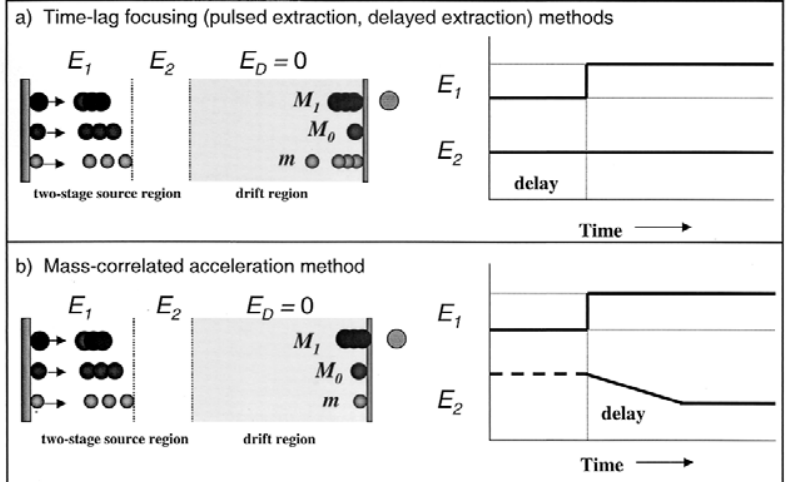

Figure 1. Time-lag focusing extraction methods: (a) The first extraction field $E_{1}$ is pulsed, a delay time and/or pulse amplitude are mass dependent, and a narrow mass range $\left(M_{0}\right)$ is focused. $(\mathbf{b})$ Mass correlated acceleration: A time dependent field $E_{2}$ is added to the second extraction region so that ions of mass $m<M_{0}$ are focused, and the method is mass-independent over a broad mass range.

stage [16] ion extraction regions in a linear MALDI TOF mass spectrometer. The detailed mathematical treatment and experimental verification of the method was reported [17] which showed isotopic mass resolution over the mass range from 901.14542.1 Da. The approach was based on the assumption that within the second region of the dual-stage ion source, a correctly defined time-dependent voltage function satisfies first-order focusing conditions over the entire mass range of relevance. To provide focusing over a broad mass range, each isomass packet is treated separately. This is plausible since it is known that ions of different masses (after pulsed extraction) enter the acceleration region at different times. To shift the focal point farther away from the source and closer to the detector for ions $m<M_{0}$ in the same isomass ion packet, the leading ions need less acceleration while the trailing ions need more acceleration. Thus, the correction voltage should begin with a positive value, decrease with time and disappear at the time the $M_{0}$ ions enter the acceleration region.

Figures $1 \mathrm{a}$ and $\mathrm{b}$ compare time-lag focusing with MCA. In Figure 1a the first extraction field $\left(E_{1}\right)$ is pulsed, and only a narrow ion mass range $\left(M_{0}\right)$ is focused at a given delay time and/or pulse amplitude. The lighter ions, $m$, focus before the detector while the heavy ions, $M_{1}$, focus behind the detector. In Figure $1 \mathrm{~b}$ a time dependent extraction field $\left(E_{2}\right)$ is added to the second region so that ions of mass $m<M_{0}$ (where $M_{0}$ is a reference mass) are focused. Operationally, the time delay is set to focus on the highest mass in the range to be focused, and then all lighter ions focus at the detector as well. Higher mass resolution is demonstrated over a broad mass range under a single set of tuning parameters such as extraction voltage, delay time, laser power, and pulse amplitude.

While MCA improves the mass range over which ions are focused in a linear MALDI TOF [17], we have now modified the instrument by incorporating a reflectron to enhance the absolute mass resolution over that range. The reflectron is a post-source focusing method while techniques such as PE and MCA are in-source focusing methods. Both techniques lead to improved resolution. Mamyrin first introduced the reflectron, or ion mirror, in 1973 [18], which compensated for the initial kinetic energy distribution by ensuring that ions of the same mass but with different kinetic energies simultaneously arrive at the detector. This requires that isomass ions of different energy are at the focal point for the reflectron at the same time.

The calculation of optimal experimental parameters for carrying out MCA in a reflectron (re)-MALDI TOF mass spectrometer are the same as previously described for a linear instrument, with the addition of another term, $t_{R}$, into eq 9 in that report [17] to account for the time that an ion spends in the reflectron part of the analyzer:

$$
\mathrm{t}_{\mathrm{R}}=2 v_{\mathrm{B}} d_{\mathrm{R}} m / e \mathrm{U}_{\mathrm{R}}
$$

where $U_{R}$ is the voltage applied across the reflector of length $d_{R}$, and $v_{\mathrm{B}}$ is the velocity of an ion with mass $m$ upon leaving the acceleration region where the correction waveform is applied. The optimum delay times for a reference mass are then calculated for a reflectron configuration. In addition, the geometry of the modified reflectron instrument varies from the linear TOF mass spectrometer previously reported. In the reflectron (re)TOF instrument, the length of the first extraction region, $0.36 \mathrm{~cm}$, is the same as in the linear instrument, while the acceleration region is $3.10 \mathrm{~cm}$, or $1.36 \mathrm{~cm}$ shorter than the original length in the linear instrument, reflecting the shorter distance from the source that locates the focal point for the reflectron.

Because the value of the initial velocity is known only approximately, the optimal correction voltage waveform that would be applied to the acceleration region for different values of ion initial velocities was plotted (Figure 2). Variations in $v_{\mathrm{o}}$ result in changes in both delay time for the extraction pulse and $\mathrm{U} / \mathrm{U}_{0}$. It was previously shown that the choice of acceleration region length must achieve a compromise between the lowest possible high voltage (HV) correction pulse amplitude and a practical electronic circuit capable of giving an adequate pulse waveform [17]. The acceleration region is longer than in conventional PE experiments to allow for the simplest correction pulse shape over the widest mass range possible. It must be noted, however, that any MALDI instrument employing PE can be modified to improve mass resolution over a broad mass range as different acceleration region lengths can be used with appropriate correction pulse waveforms. However, the MCA method described here attempts to optimize the extraction geometry to provide optimal mass-independent focusing. 


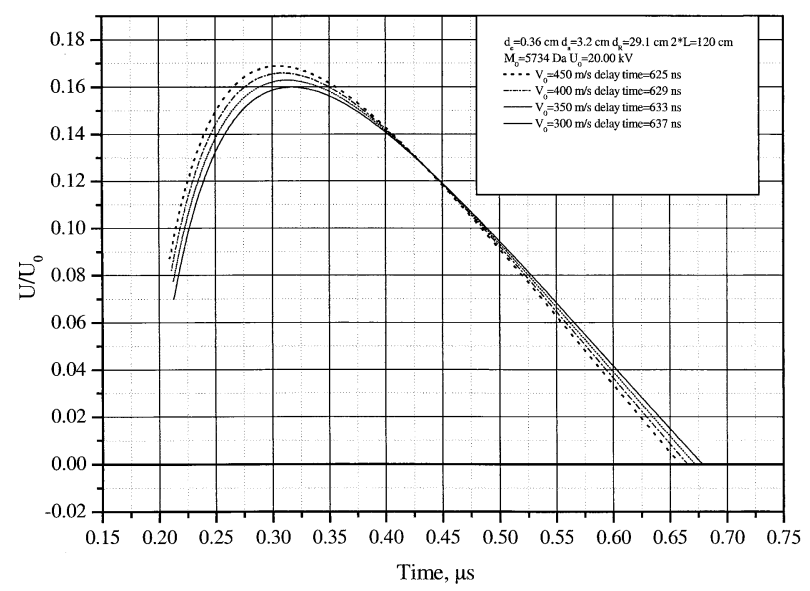

Figure 2. Correction pulse waveform showing the optimal correction voltage waveform $\left(\mathrm{U} / \mathrm{U}_{0}\right)$ that would be applied to the acceleration region $\left(d_{\mathrm{a}}\right)$ for different values of ion initial velocities $\left(v_{\mathrm{o}}\right)$. Other parameters include the extraction region $\left(d_{\mathrm{e}}\right)$, distance of the reflectron $\left(d_{\mathrm{r}}\right)$, and the reference mass $\left(M_{0}\right)$.

\section{Experimental}

\section{Instrument}

The linear MALDI TOF mass spectrometer that was built in-house and described elsewhere [17] was modified with a reflectron (re) to further improve mass resolution over a broad mass range. Figure 3 is a schematic of the re-MALDI TOF mass spectrometer. The vacuum chamber is pumped via a Turbovac 360 CSV turbopump (Leybold Vacuum Products, Export, $\mathrm{PA}$ ) with the pressure in the TOF region maintained below $5 \times 10^{-7}$ torr. A pulsed nitrogen laser VSl337ND-S 1 (Laser Science, Franklin, MA) is capable of delivering $300 \mu \mathrm{J}$ energy and a $<4$ ns short width pulse (peak power approximately $75 \mathrm{~kW}$ ). The laser beam is directed by a flat mirror 2, an iris diaphragm 3, a variable optical density filter 4 , and focused on the probe tip target 5 by a UV Bestform lens (Special Optics, Wharton, JH) (75 mm focal length) 6 situated outside the chamber. All spectra are recorded at irradiances $10-15 \%$ above threshold. The angle of incidence measures $60^{\circ}$ relative to the sample surface normal. A CDC digital camera images the laser spot area (approximately $60 \mu \mathrm{m}^{2}$ ). A pulse generator BNC 8010 (Berkely Nucleonics, Berkeley, CA) 7 externally triggers the laser. A trigger TTL signal from the low-jitter ( $<1 \mathrm{~ns})$ laser output drives a pulse generator DG535 (Stanford Research Systems, Sunnyvale, CA) 8 providing timing for HV pulses and data recording. The pulse generator delivers TTL pulses ( 3 ns rise time) to the Lecroy $9374 \mathrm{M}$ oscilloscope 9 (500 MHz, $2 \mathrm{Gs} / \mathrm{s}$ acquisition rate), the fast HV switch (HTS 30-06, Eurotech, Morganville, NJ) 10, and a correction pulse generator 11 built in-house.

Grid 112 separates the extraction region from the acceleration region and is biased at $17.157 \mathrm{kV}$ by a $\mathrm{HV}$ power supply 13 (model 612C, Bertan, Hicksville, NY). An extraction plate 14 receives the same voltage via a resistor $\mathrm{R} 2$ of $5 \mathrm{M} \mathrm{ohm}$. The extraction plate is pulsed by a fast HV switch $\mathbf{1 0}(<20 \mathrm{~ns}$ rising edge time) with an optimized delay time for a selected mass $M_{0}$ (corresponding to the high mass end of the range). The switch $\mathbf{1 0}$ delivers a pulse to an electrode $\mathbf{1 5}$ via a coupling low-inductance capacitor $\mathrm{C} 1$ of $1000 \mathrm{pF}$ and resistor R1 of $100 \mathrm{ohms}$. This switch $\mathbf{1 0}$ is biased by a power supply PS350 (Stanford Research Systems). A ceramic lowinductance capacitor C2 of $1500 \mathrm{pF}$ is used to suppress

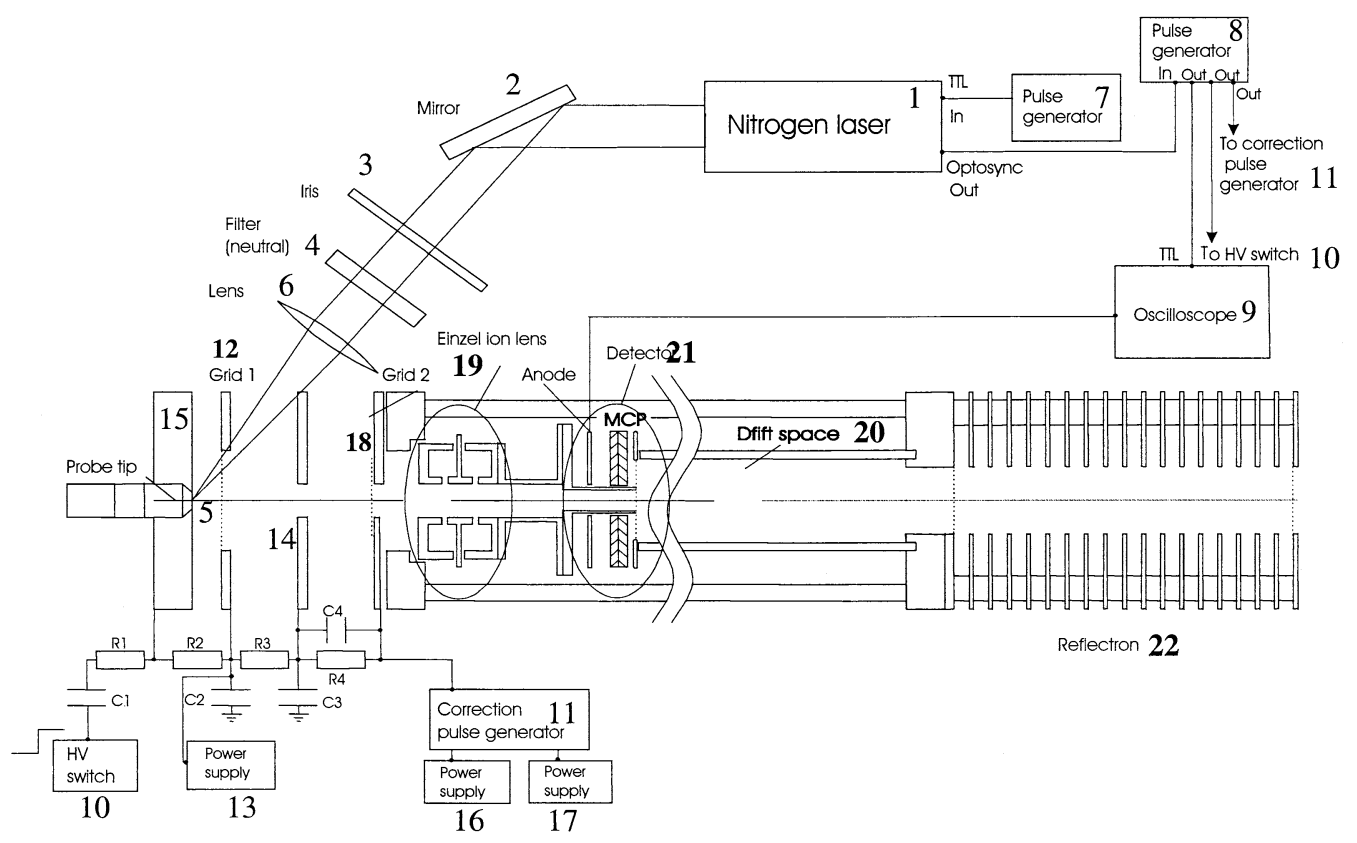

Figure 3. Schematic of re-MALDI TOF mass spectrometer equipped with a dual-stage ion source utilizing mass correlated acceleration (MCA). 


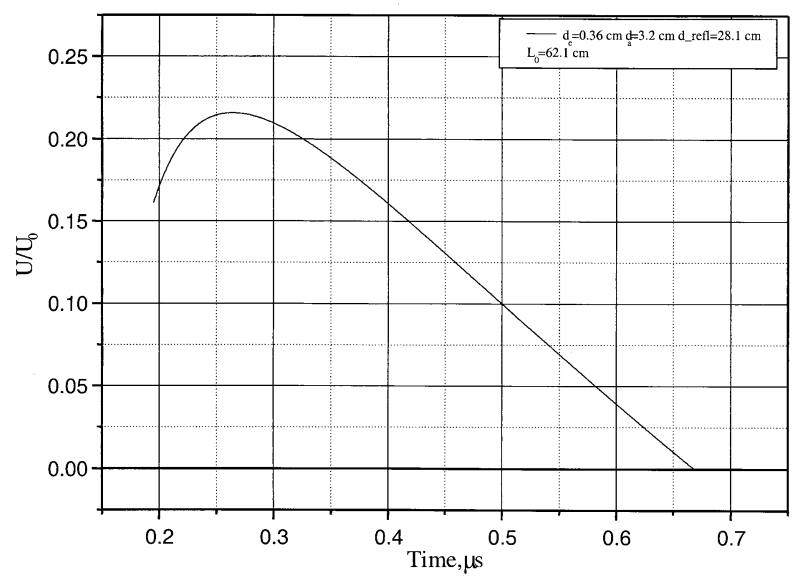

Figure 4. The correction pulse waveform used in mass correlated acceleration for the re-MALDI TOF mass spectrometer.

the effects of the flyback voltage resulting from capacitive coupling.

Once optimum resolution is obtained for the highest mass ion by pulsed extraction in the first region only, the additional acceleration region is turned on to focus all ions $m<M_{0}$. The correction pulse waveform is produced by the fast HV switch in the correction pulse generator 11. This bell-shaped waveform, as seen in Figure 4, is produced by splicing opposing rising and falling waveforms where the low voltage level of $-5.790 \mathrm{kV}$ is provided by a power supply 16, PS350 (Stanford Research Systems), and the upper voltage level of $+6.000 \mathrm{kV}$ is provided by a power supply $\mathbf{1 7}$, model 320P (Bertan, Hicksville, NY). High-frequency capacitors C3, C4, and C5 (in series) establish an even correction voltage distribution across the $3.10 \mathrm{~cm}$ acceleration region.

Grid 218 separates the acceleration region from the Einzel lens 19 and drift space 20 regions. The Einzel lens is biased and optimized between 8.25 and $8.50 \mathrm{kV}$ by a power supply, model 320P (Bertan, Hicksville, NY). The ions are focused through the center hole $(6 \mathrm{~mm}$ diameter) of a microchannel plate (MCP) coaxial detector $\mathbf{2 1}$ by an Einzel lens and into the field-free drift region 20. The total ion drift length is $120.2 \mathrm{~cm}$. The ions then enter a reflectron 22 biased at $22.0 \mathrm{kV}$ by a power supply, model 205B (Bertan, Hicksville, NY). The reflectron is positioned at the end of the drift tube and measures 29.1 $\mathrm{cm}$ in length. The assembly contains a stack of 7.0 by 7.0 $\mathrm{cm}$ rectangular plates with a $40 \mathrm{~mm}$ central hole. The plates are separated by ceramic spacers, each measuring $6.43 \mathrm{~mm}$ long. Upon exiting the reflectron and passing back through the drift region, the ion signal is registered at a detector. A coaxial Hamamatsu MCP detector (model F4294-09) 21 with a $6 \mathrm{~mm}$ central hole is used for ion detection. The detector is biased at $-2400 \mathrm{~V}$ by a power supply, model SRS PS350 (Stanford Research Systems).

Spectra are averaged from 50-100 laser shots on the same sample spot. The ion signal is monitored with an oscilloscope 9 previously described. Delay times include $640 \mathrm{~ns}$ for the extraction pulse; an additional 130 ns delay via a pulse generator, model DG525 (Stanford Research Systems) for the negative-going part of the correction pulse; and an additional 110 ns delay for the positive-going part of the correction pulse via a pulse generator, model DG525 (Stanford Research Systems). Data are transferred and stored in a personal computer using commercial software (TOFWARE, Ilys Software, Pittsburgh, PA).

\section{Materials}

The following peptides were purchased from Sigma Chemical (St. Louis, MO) and used without further purification: Bradykinin fragment 1-7, des-Arg ${ }^{9}$-bradykinin, substance $\mathrm{P}$, neurotensin, dynorphin $\mathrm{A}$, somatostatin 28, adrenocorticotropic hormone, fragment 7-38 (ACTH 7-38), ACTH 1-39, and bovine insulin. The Milli-Q water was purified with a Millipore deionization system (Bedford, MA). Ethanol and methanol were HPLC grade and purchased from J. T. Baker (Phillipsburg, NJ). Trifluoroacetic acid (TFA) was purchased from Aldrich (Milwaukee, WI). Trypsin was purchased from Roche Molecular Biochemicals (Berkeley, CA). The proteins lysozyme $(14,307.2 \mathrm{Da})$ and bovine serum albumin $(66,430.09 \mathrm{Da})$ were purchased from Sigma Chemical (St. Louis, MO) and used as received. $\alpha$-cyano-4-hydroxycinnamic acid was purchased from Aldrich (Milwaukee, WI) and used as received. Ziptip pipets were purchased from Millipore (Bedford, MA) and used for protein digest purification. Theoretical monoisotopic molecular weights for fragment ions resulting from tryptic digests were found on Swiss-Prot (Protein Knowledgebase) at the website http:// www.expasy.ch/sprot/sprot-top.html.

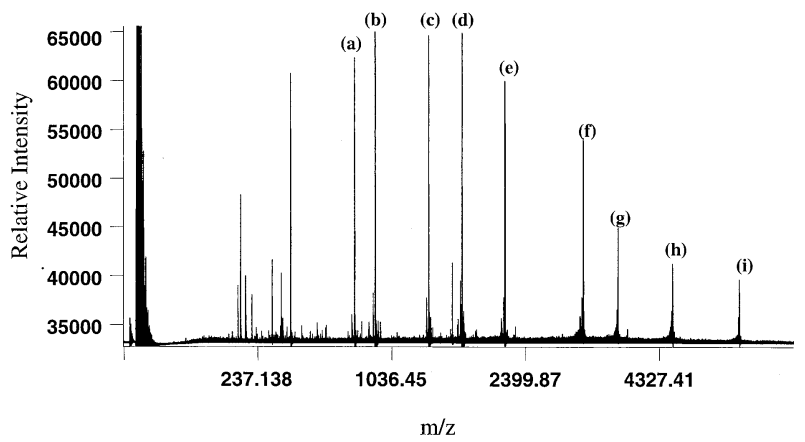

Figure 5. Mass spectrum (averaged) of a nine-peptide mixture obtained with mass-correlated acceleration in a re-MALDI TOF mass spectrometer. Peptides and corresponding monoisotopic (M $+\mathrm{H})^{+}$values include (a) bradykinin, fragment 1-7 (757.40 Da); (b) des-Arg'-bradykinin (904.47 Da); (c) substance P (1347.74 Da); (d) neurotensin (1672.92 Da); (e) dynorphin A (2147.20 Da); (f) somatostatin 28 (3147.47 Da); (g) adrenocorticotropic hormone, fragment 7-38 (ACTH 7-38) (3657.93 Da); (h) ACTH 1-39 (4539.27 Da); (i) bovine insulin $(5730.61 \mathrm{Da})$. 
(a) Bradykinin 1-7

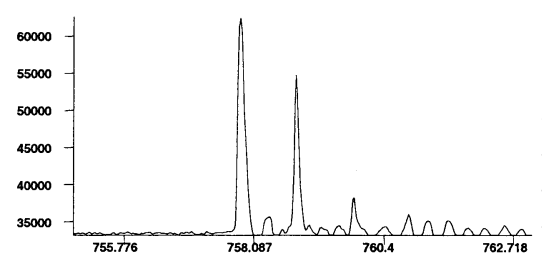

(d) Neurotensin

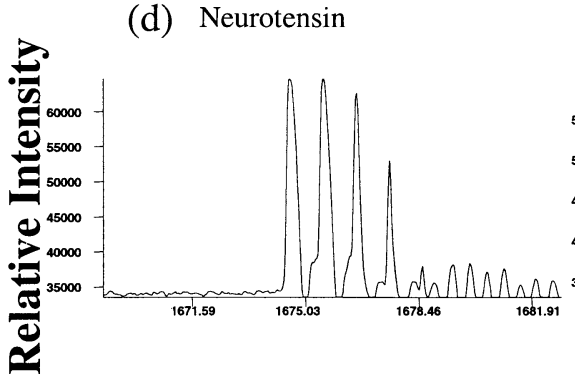

(g) $\quad$ ACTH 7-38

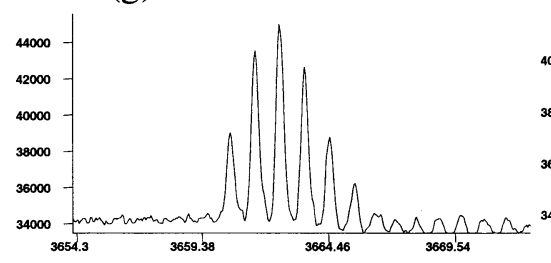

(b) des-Arg'-bradykinin

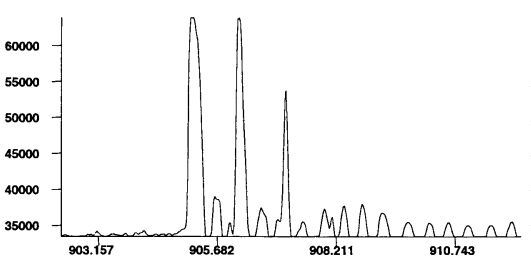

(e) Dynorphin A (c) Substance P

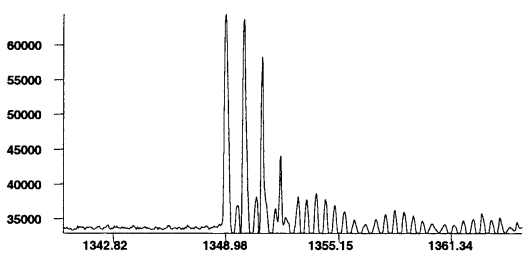

(f) Somatostatin 28
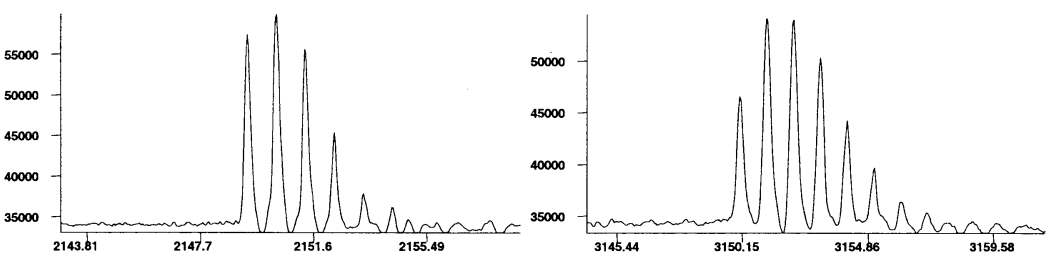

(h) $\quad$ ACTH 1-39

(i) Bovine insulin
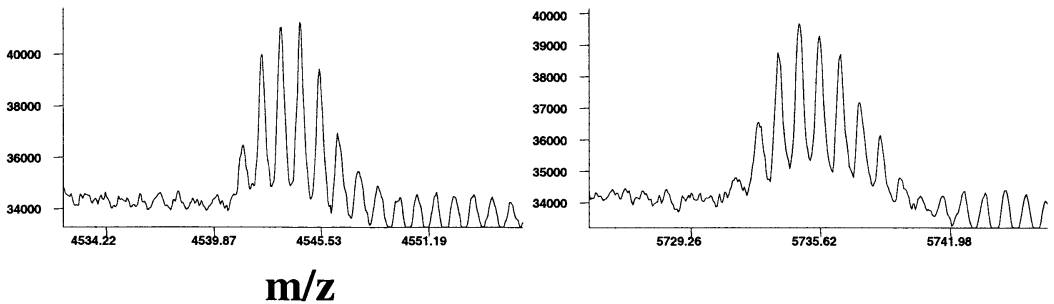

Figure 6. Molecular ion regions for the peptides shown in Figure 5 in mass correlated acceleration mode.

\section{Sample Preparation}

Peptide mix experiments. Stock solutions of all the peptides were prepared to concentrations of $400 \mathrm{pmol} / \mu \mathrm{L}$ in $0.1 \%$ TFA. The molar amounts of peptides on the probe were as follows: Bradykinin, fragment 1-7 (1.6 pmol), des-Arg'-bradykinin (1.6 pmol), neurotensin (1.9 pmol), substance P (1.9 pmol), dynorphin A (2.9 pmol), somatostatin $28(3.0 \mathrm{pmol})$, ACTH 7-38 (3.6 pmol), ACTH 1-39 (3.6 pmol) and bovine insulin (4.0 pmol). A saturated solution of $\alpha$-cyano-4-hydroxycinnamic acid was prepared in 1:1 (vol:vol) 0.1\%:ethanol.

The probe tip surface was first polished with 3600 grade finishing paper and then cleaned with ethanol and water. Sample preparation on the probe was derived from the method described [19] and was designed to minimize initial spatial distribution by reducing the crystal size. $0.5 \mu \mathrm{L}$ of matrix solution was deposited on the probe tip and allowed to dry. A glass slide was placed over the probe tip, matrix was crushed by application of pressure, and excess matrix particles were removed. Next, $0.5 \mu \mathrm{L}$ of a solution (2:1 (vol:vol) matrix:peptide mixture, centrifuged for $4 \mathrm{~min}$ to remove undissolved matrix particles) was placed on the top of the smeared matrix layer and allowed to dry. The sample was washed as follows: 2-3 times with $5 \mu \mathrm{L}$ ice-cold $0.1 \%$ TFA placed on the top and removed approximately $5 \mathrm{~s}$ later using a gentle stream of air. The probe tip was then inserted into the mass spectrometer.

Protein digest experiments. The following protocols were followed. Lysozyme: Stock solutions were prepared of lysozyme $(5 \mathrm{mg} / \mathrm{mL}$ in water), trypsin $(1.25$ $\mathrm{mg} / \mathrm{mL}$ in $1 \%$ acetic acid), and tris $\mathrm{HCl}(100 \mathrm{mM}$ in $1 \%$ $\beta$-mecaptoethanol at $\mathrm{pH}=8.5) .50 \mu \mathrm{L}$ tris $\mathrm{HCl}, 25 \mu \mathrm{L}$ lysozyme, and $1.75 \mu \mathrm{L}$ trypsin were mixed in a microcentrifuge tube and reacted for $18 \mathrm{~h}$ at $37^{\circ} \mathrm{C}$. Bovine

Table 1. Components of a peptide mixture analyzed in a MCA re-MALDI TOF mass spectrometer

\begin{tabular}{lccc}
\hline Peptide name & $\begin{array}{c}\text { Monoisotopic } \\
(\mathrm{M}+\mathrm{H})^{+}\end{array}$ & $\begin{array}{c}\text { Peak } \\
\text { width (ns) }\end{array}$ & $\begin{array}{c}\text { Mass } \\
\text { resolution }\end{array}$ \\
\hline \hline $\begin{array}{l}\text { Bradykinin, frag. } \\
\quad 1-7\end{array}$ & 757.40 & 2 & 8395 \\
$\begin{array}{l}\text { des-Arg }{ }^{9} \\
\text { bradykinin }\end{array}$ & 904.47 & 2 & 9154 \\
$\begin{array}{l}\text { Substance P } \\
\text { Neurotensin }\end{array}$ & 1347.74 & 2 & 11124 \\
Dynorphin A & 1672.92 & 3 & 8245 \\
Somatostatin 28 & 2147.20 & 3 & 9323 \\
ACTH 7-38 & 3147.47 & 3 & 11257 \\
ACTH 1-39 & 3657.93 & 3 & 12120 \\
Insulin (bovine) & 4539.27 & 3 & 13487 \\
\hline
\end{tabular}


(a) Bradykinin 1-7

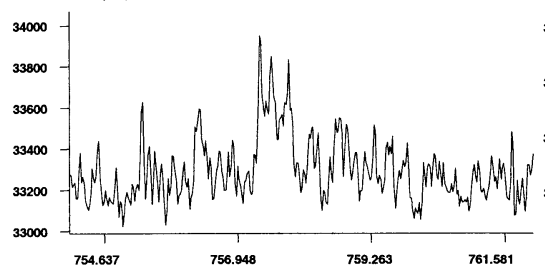

(d) Neurotensin

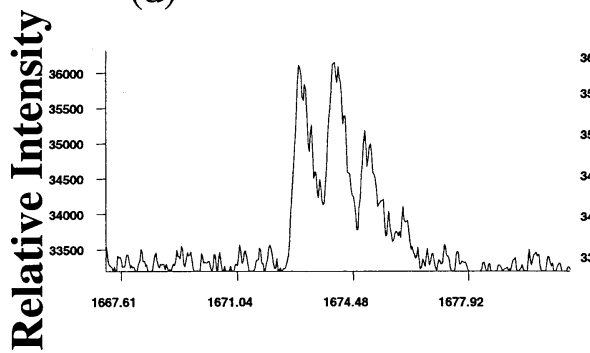

(g) $\mathrm{ACTH} 7-38$ (b) des-Arg' ${ }^{9}$-bradykinin

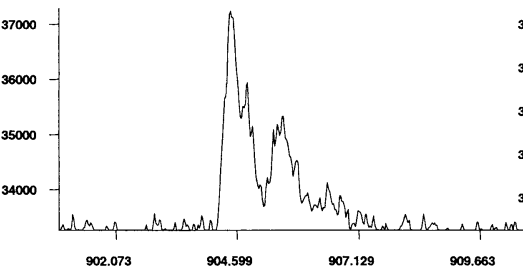

(e) Dynorphin A (c) Substance P

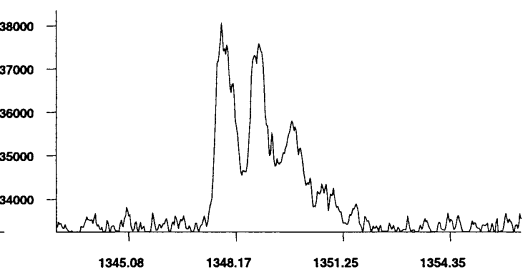

(f) Somatostatin 28

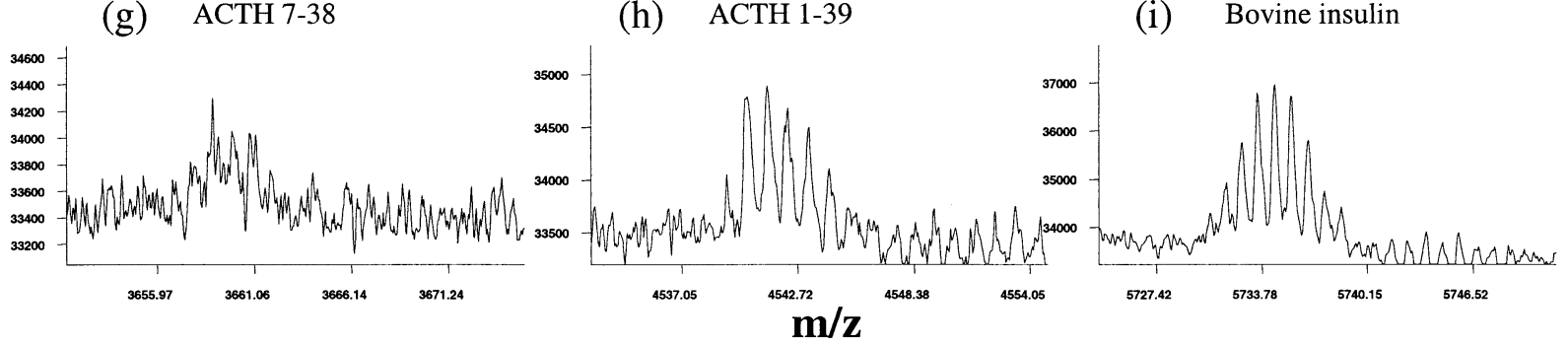

Figure 7. Molecular ion regions for the peptides in pulsed extraction mode.

serum albumin (BSA): Stock solutions were prepared of BSA $(5 \mathrm{mg} / \mathrm{mL}$ in water), trypsin $(1.25 \mathrm{mg} / \mathrm{mL}$ in $1 \%$ acetic acid), and ammonium bicarbonate buffer (50 $\mathrm{mM}) .36 \mu \mathrm{L}$ ammonium bicarbonate buffer, $18 \mu \mathrm{L}$ BSA, and $3 \mu \mathrm{L}$ trypsin were mixed in a microcentrifuge tube and reacted for $18 \mathrm{~h}$ at $37^{\circ} \mathrm{C}$. After reacting for $18 \mathrm{~h}$, both the Lys and BSA digests were removed and purified with a standard C18 Ziptip protein cleanup protocol supplied by the manufacturer.

The MALDI probe tip was polished with 8000 and 12,000 grade finishing paper and then cleaned with ethanol and water. Three different sample spots were deposited onto the MALDI probe tip. The first spot consisted of $0.5 \mu \mathrm{L} \alpha$-cyano-4-hydroxycinnamic acid (saturated solution in 2:2:1 (vol:vol:vol) water:ethanol: methanol) and $0.5 \mu \mathrm{L}$ purified lysozyme mixed on the sample probe tip. The second spot consisted of $0.5 \mu \mathrm{L}$ $\alpha$-cyano-4-hydroxycinnamic acid and $0.5 \mu \mathrm{L}$ purified BSA mixed on the sample probe tip. To act as an internal calibrant, a third sample was deposited that contained $0.5 \mu \mathrm{L} \alpha$-cyano-4-hydroxycinnamic acid and $0.5 \mu \mathrm{L}$ of a peptide mixture. The molar amounts of the peptides on the probe tip were as follows: des-Arg ${ }^{9}$ bradykinin, 22 pmol; neurotensin, 22 pmol; dynorphin A, 11 pmol; insulin chain B, 32 pmol; ACTH 1-39, 22 pmol; bovine insulin, 11 pmol. The peptide mix spot was then washed with $2 \mu \mathrm{L}$ of cold $0.1 \%$ TFA, and the samples were allowed to dry in air. The probe tip was briefly blown dry with a gentle stream of air and inserted into the mass spectrometer.

\section{Results}

MALDI is a powerful technique for the analysis of mixtures, for it has become widely used to analyze protein mixtures from biological samples $[20,21]$ and to sequence oligonucleotide mixtures [22]. When analyzing mixtures with a wide dispersion in molecular weight of its components, a MALDI instrument which offers high mass resolution over the entire mass range in a single experiment (under a single set of tuning parameters) would be ideal. We show data from peptide mixtures and protein digests that support these types of applications.

Figure 5 shows a mass spectrum of nine peptides obtained by MCA in a re-MALDI TOF mass spectrometer. The instrument is tuned to focus on the highest mass, $M_{0}$, which in this case is the $(\mathrm{M}+\mathrm{H})^{+}$monoisotopic peak at $5730.61 \mathrm{Da}$ for bovine insulin. The time dependent waveform in the acceleration region focuses all ions $m<M_{0}$ with improved resolution across the entire mass range studied as shown for the molecular 


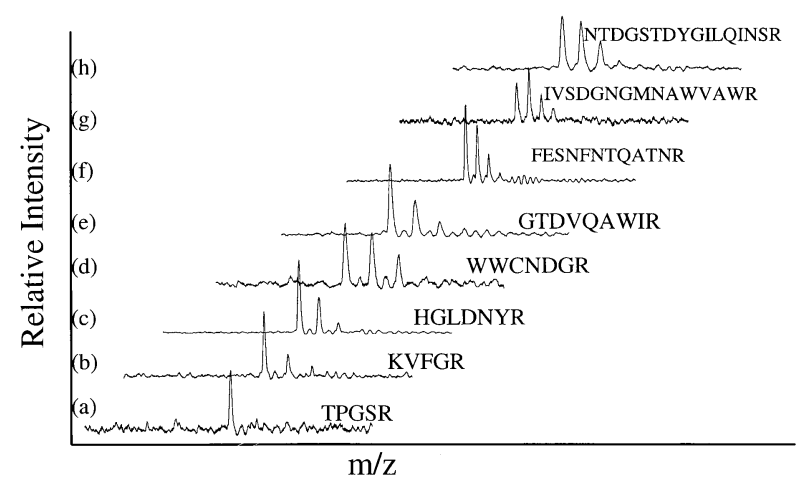

Figure 8. Monoisotopic $(\mathrm{M}+\mathrm{H})^{+}$peak profiles in a mass spectrum (averaged) of lysozyme tryptic digest fragments obtained with mass-correlated acceleration in a re-MALDI TOF mass spectrometer: (a) TPGSR (517.27 Da), (b) KVFGR (606.37 Da), (c) HGLDNYR (874.42 Da), (d) WWCNDGR (936.38 Da), (e) GTDVQAWIR (1045.54 Da), (f) FESNFNTQATNR (1428.65 Da), (g) IVSDGNGMNAWVAWR (1675.80 Da), (h) NTDGSTDYGILQINSR (1753.84 Da).

ion regions depicted in Figure 6. Isotopic resolution is observed across the entire mass range from bradykinin to bovine insulin. Table 1 lists the peak widths and mass resolution for the peptides in the mixture. Resolution was calculated as $\mathrm{t} / 2 \Delta \mathrm{t}$ where $\mathrm{t}=$ ion flight time and $\Delta t$ is calculated at FWHM.

For comparison, the instrument was then operated in conventional pulsed extraction mode (i.e., the voltage applied to the second region was turned off). The spectra in Figure 7 demonstrate that (compared to mass correlated acceleration) pulsed extraction does not supply the enhanced resolution over the entire mass range studied. The resolution is better towards the upper mass range since the largest mass (5730.61 Da) was being focused. Resolution decreases for all other masses below this reference mass. However, it must be noted that the second region in the current instrument is longer than in typical pulsed extraction instruments to lengthen the time spent within the time-varying field. This was also the case for the linear instrument described previously [17], and makes the comparison with normal pulsed methods less than ideal.

While MCA re-MALDI TOF focuses ions of a broad

Table 2. Lysozyme tryptic digest analyzed in a re-MALDI TOF mass spectrometer

\begin{tabular}{lccc}
\hline $\begin{array}{l}\text { Fragment ion } \\
\text { sequence }\end{array}$ & $\begin{array}{c}\text { Monoisotopic } \\
(\mathrm{M}+\mathrm{H})^{+}\end{array}$ & $\begin{array}{c}\text { Peak } \\
\text { width (ns) }\end{array}$ & $\begin{array}{c}\text { Mass } \\
\text { resolution }\end{array}$ \\
\hline \hline TPGSR & 517.27 & 3 & 4693 \\
KVFGR & 606.37 & 3 & 5067 \\
HGLDNYR & 874.42 & 3 & 6049 \\
WWCNDGR & 936.38 & 3 & 6254 \\
GTDVQAWIR & 1045.54 & 3 & 6598 \\
FESNFNTQATNR & 1428.65 & 3 & 7684 \\
IVSDGNGMNAWVAWR & 1675.80 & 3 & 8310 \\
NTDGSTDYGILQINSR & 1753.84 & 3 & 8495 \\
\hline
\end{tabular}

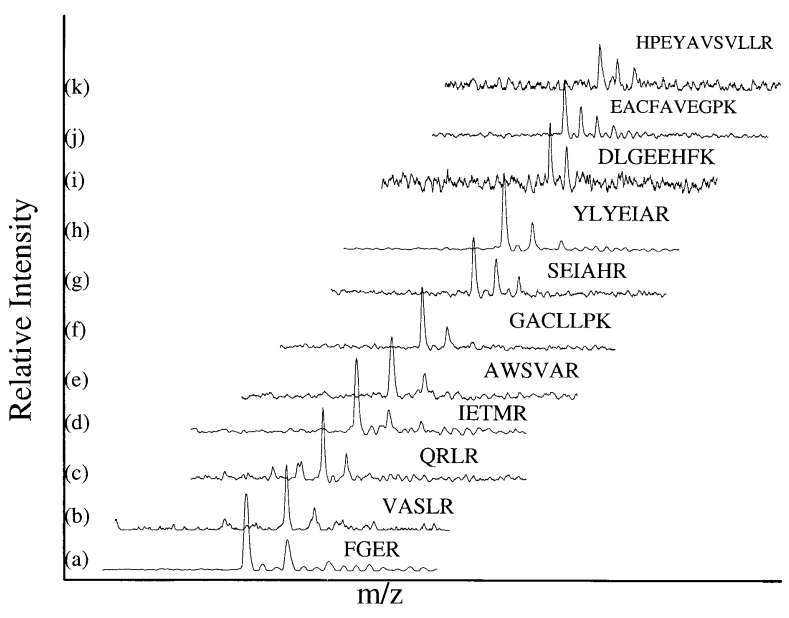

Figure 9. Monoisotopic $(\mathrm{M}+\mathrm{H})^{+}$peak profiles in a mass spectrum (averaged) of BSA tryptic digest fragments obtained with mass-correlated acceleration in a re-MALDI TOF mass spectrometer: (a) FGER (508.25 Da), (b) VASLR (545.34 Da), (c) QRLR (572.36 Da), (d) IETMR (649.33 Da), (e) AWSVAR (689.37 Da), (f) GACLLPK (701.40 Da), (g) SEIAHR (712.37 Da), (h) YLYEIAR $(927.49 \mathrm{Da}),(\mathbf{i})$ DLGEEHFK $(974.46 \mathrm{Da}),(\mathbf{j})$ EACFAVEGPK (1050.49 Da), (k) HPEYAVSVLLR (1283.71 Da).

mass range in peptide mixtures, we also demonstrated its focusing capabilities with other biological samples such as protein digests. Protein digests offer a means to fragment a protein so that the smaller pieces can be identified, leading to possible protein sequence information. This is a widely used method for sequencing proteins in the field of proteomics. Subsequently, the probe tip was loaded with three sample spots using $\alpha$-cyano-4-hydroxycinnamic acid as the matrix: Lysozyme digest, BSA digest, and the peptide mix (for calibration). Figure 8 displays the peak profiles obtained from a tryptic digest of the protein lysozyme $(14,307.2 \mathrm{Da})$ in a single MALDI experiment. One set of tuning parameters was used and spectra were averaged from 100 laser shots on a single sample spot, although fewer laser shots could have been used to obtain a mass spectrum. The mass range of the fragment ions is approximately 500-1800 $\mathrm{Da}$. No ions of higher mass

Table 3. Bovine serum albumin tryptic digest analyzed in a MCA re-MALDI TOF mass spectrometer

\begin{tabular}{lccc}
$\begin{array}{l}\text { Fragment ion } \\
\text { sequence }\end{array}$ & $\begin{array}{c}\text { Monoisotopic } \\
(\mathrm{M}+\mathrm{H})^{+}\end{array}$ & $\begin{array}{c}\text { Peak } \\
\text { width }(\mathrm{ns})\end{array}$ & $\begin{array}{c}\text { Mass } \\
\text { resolution }\end{array}$ \\
\hline \hline FGER & 508.25 & 3 & 4653 \\
VASLR & 545.34 & 3 & 4813 \\
QRLR & 572.36 & 3 & 4927 \\
IETMR & 649.33 & 3 & 5237 \\
AWSVAR & 689.37 & 3 & 5391 \\
GACLLPK & 701.40 & 3 & 5436 \\
SEIAHR & 712.37 & 3 & 5477 \\
YLYEIAR & 927.49 & 3 & 6225 \\
DLGEEHFK & 974.46 & 3 & 6376 \\
EACFAVEGPK & 1050.49 & 3 & 6613 \\
HPEYAVSVLLR & 1283.71 & 3 & 7293 \\
\hline
\end{tabular}


Table 4. Comparison of two-point calibration methods

\begin{tabular}{|c|c|c|c|c|c|c|}
\hline \multirow[b]{2}{*}{ Peptide name } & \multicolumn{2}{|c|}{ Calibration 1} & \multicolumn{2}{|c|}{ Calibration 2} & \multicolumn{2}{|c|}{ Calibration 3} \\
\hline & $(\mathrm{M}+\mathrm{H})^{+}$ & $\Delta \mathrm{m}$ & $(\mathrm{M}+\mathrm{H})^{+}$ & $\Delta \mathrm{m}$ & $(\mathrm{M}+\mathrm{H})^{+}$ & $\Delta \mathrm{M}$ \\
\hline Bradykinin, frag. 1-7 & calibrant & - & 756.9 & -0.5 & 754.8 & -2.6 \\
\hline des-Arg ${ }^{9}$-bradykinin & 904.6 & +0.1 & 904.1 & -0.4 & 902.0 & -2.5 \\
\hline Substance $\mathrm{P}$ & 1348.2 & +0.5 & calibrant & - & 1345.6 & -2.1 \\
\hline Neurotensin & 1673.6 & +1.0 & 1673.2 & +0.3 & 1671.1 & +1.8 \\
\hline Dynorphin A & 2148.2 & +0.7 & 2147.8 & +0.6 & 2146.0 & -1.2 \\
\hline Somatostatin 28 & 3148.5 & +1.0 & 3148.2 & +0.7 & 3147.2 & -0.3 \\
\hline АСТН 7-38 & 3658.7 & +0.8 & 3658.6 & +0.7 & calibrant & - \\
\hline АСТН 1-39 & calibrant & - & calibrant & - & 4539.5 & +0.2 \\
\hline Insulin (bovine) & 5728.8 & -1.8 & 5729.1 & -1.5 & calibrant & - \\
\hline
\end{tabular}

were detected. A six-peptide mixture was used to calibrate the spectra for the protein digest experiments. Table 2 lists the lysozyme tryptic digest fragment ion sequence, monoisotopic $(\mathrm{M}+\mathrm{H})^{+}$value, peak width and mass resolution (FWHM) of each ion. All of the fragment ions from the tryptic digest resulted from zero missed cleavages, according to the Swiss-Prot database, with the exception of the fragment ion KVFGR which resulted from one missed cleavage.

The sample insertion rod was rotated so that the laser now focused on a BSA protein sample area. Figure 9 shows the molecular peak profiles obtained from a tryptic digest of BSA $(66,430.09 \mathrm{Da})$ in a single MALDI mass spectrum. The mass range of the tryptic digest fragment ions is approximately 500-1800. No ions of higher mass were detected. Table 3 lists the BSA tryptic digest fragment ion sequence, monoisotopic $(\mathrm{M}+\mathrm{H})^{+}$ value, peak width and resolution (FWHM) of each ion. Peak widths are $3 \mathrm{~ns}$. All of the fragment ions from the tryptic digest of BSA resulted from zero missed cleavages, according to the Swiss-Prot database, with the exception of the fragment ion QRLR which resulted from one missed cleavage. While the mass range is limited in this example, it should be noted that the ion focusing conditions (using 5730.61 Da as the reference mass) were employed.

As demonstrated in mass spectra shown in this paper, the peak widths do not exceed 3 ns at FWHM using MCA, and mass resolution is improved dramatically across a broad mass range. These results support previous experiments with peak widths of 2-3 ns in MCA mode compared to 3-24 ns in PE mode for a peptide mixture [17].

Mass calibration in the re-MALDI TOF mass spectrometer with MCA is performed with a known peptide mixture discussed in the Experimental section. Table 4 lists the three calibration procedures used including the measured $(\mathrm{M}+\mathrm{H})^{+}$values and $\Delta \mathrm{m}$ to assess the mass accuracy of mass correlated acceleration in a reflectron MALDI-TOF instrument. Initially, a two-point calibration was used over the entire $(\mathrm{M}+\mathrm{H})^{+}$monoisotopic mass range 757.40-5730.61 Da. As can be seen in Table 4 (Calibration 2), using the molecular ion peaks of substance P (1347.74 Da) and ACTH 1-39 (4539.27 Da) gave the best accuracy over the given mass range with a maximum deviation of $1.5 \mathrm{Da}$ for bovine insulin. Because the final kinetic energies of ions (after acceleration) vary with mass, accurate mass assignment over a wide range requires several calibration points. Algorithms for such an approach are being developed [23].

\section{Conclusion}

In summary, the MCA method employed in a reflectron MALDI TOF mass spectrometer provides enhanced resolution over a very broad mass range. In the traditional pulsed extraction (PE) mode adjustment of the delay time and/or extraction voltage is required to achieve high mass resolution over a range of masses. Our method, however, allows for a single set of parameters to more fully achieve the multi-channel recording advantage of TOF. We demonstrated peak widths not exceeding $3 \mathrm{~ns}$ for ions varying in mass from $<1000$ to $>5000$ for peptide mixtures and protein digests. This dual-stage, mass independent ion source which focuses ions over a broad mass range is applicable to biological samples ranging from mixtures of peptides (or proteins, oligonucleotides, polymers, etc.) to protein digestions with various fragments ranging in molecular weight.

\section{Acknowledgments}

The authors are grateful to Suzanne M. Ramirez for her initial assistance in the protein digest and cleanup procedures. This work was supported by the Defense Advanced Research Project Agency (DARPA) (Grant DABT63-99-1-0006).

\section{References}

1. Karas, M.; Bachmann, D.; Bahr, U.; Hillenkamp, F. MatrixAssisted Ultraviolet Laser Desorption of Non-Volatile Compounds. Int. J. Mass Spectrom. Ion Processes 1987, 78, 53-68.

2. Tanaka, K., Ido, Y., Akita, S. Proceedings of the 2nd Japan-China Joint Symposium on Mass Spectrometry; Matsuda, H.; Liang, X.-T., Eds.; Osaka, 1987; pp 185-188.

3. Wiley, W. C.; McLaren, I. H. Time-of-Flight Mass Spectrometer with Improved Resolution. Rev. Sci. Instrum. 1955, 26, 1150-1157.

4. Colby, S. M.; King, T. B.; Reilly, J. P. Improving the Resolution of Matrix-assisted Laser Desorption/Ionization Time-of-flight Mass Spectrometry by Exploiting the Correlation between Ion 
Position and Velocity. Rapid Commun. Mass Spectrom. 1994, 8, 865-868.

5. Brown, R. S.; Lennon, J. J. Mass Resolution Improvement by Incorporation of Pulsed Ion Extraction in a Matrix-Assisted Laser Desorption/Ionization Linear Time-of-Flight Mass Spectrometer. Anal. Chem. 1995, 67, 1998-2003.

6. Vestal, M. L.; Juhasz, P.; Martin, S. A. Delayed Extraction Matrix-Assisted Laser Desorption Time-of-Flight Mass Spectrometry. Rapid Commun. Mass Spectrom. 1995, 9, 1044-1050.

7. Whittal, R. M.; Li, L. High-Resolution Matrix-Assisted Laser Desorption/Ionization in a Linear Time-of-Flight Mass Spectrometer. Anal. Chem. 1995, 67, 1950-1954.

8. Vestal, M. L.; Juhasz, P. Resolution and Mass Accuracy in Matrix-Assisted Laser Desorption Ionization-Time-of-Flight. J. Am. Soc. Mass Spectrom. 1998, 9, 892-911.

9. Whittal, R. M.; Russon, L. M.; Weinberger, S. R.; Li, L. Functional Wave Time-Lag Focusing Matrix-Assisted Laser Desorption/Ionization in a Linear Time-of-Flight Mass Spectrometer: Improved Mass Accuracy. Anal. Chem. 1997, 69, 2147-2153.

10. Franzen, J. Improved Resolution for MALDI-TOF Mass Spectrometers: A Mathematical Study. Int. J. Mass Spectrom. Ion Processes 1997, 164, 19-34.

11. Muga, M. L. U.S. Patent 4,458,149, July 3, 1984.

12. Yefchak, G. E.; Enke, C. G.; Holland, J. F. Models for MassIndependent Space and Energy Focusing in Time-of-Flight Mass Spectrometry. Int. J. Mass Spectrom. Ion Processes 1989, 87, 313-330.

13. Kinsel, G. R.; Johnston, M. V. Post Source Pulse Focusing: A Simple Method to Achieve Improved Resolution in a Time-ofFlight Mass Spectrometer. Int. J. Mass Spectrom. Ion Processes 1989, 91, 157-176.
14. Kinsel, G. R.; Grundwuermer, J. M.; Grotemeyer, J. J. Am. Soc. Mass Spectrom. 1993, 4, 2-10.

15. Kovtoun, S. V. An Approach to the Design of Mass-correlated Delayed Extraction in a Linear Time-of-flight Mass Spectrometer. Rapid Commun. Mass Spectrom. 1997, 11, 433-436.

16. Kovtoun, S. V. Mass-correlated Delayed Extraction in Linear Time-of-Flight Mass Spectrometers. Rapid Commun. Mass Spectrom. 1997, 11, 810-815.

17. Kovtoun, S. V.; Cotter, R. J. Mass-Correlated Pulsed Extraction: Theoretical Analysis and Implementation With a Linear Matrix-Assisted Laser Desorption/Ionization Time of Flight Mass Spectrometer. J. Am. Soc. Mass Spectrom. 2000, 11, 841-853.

18. Mamyrin, B. A.; Karataev, V. I.; Shmikk, D. V.; Zagulin, V. A. Sov. Phys. JETP 1973, 37, 45.

19. Xiang, F.; Beavis, R. C. A Method to Increase Contaminant Tolerance in Protein Matrix-assisted Laser Desorption/Ionization by the Fabrication of Thin Protein-doped Polycrystalline Films. Rapid Commun. Mass Spectrom. 1994, 8, 199-204.

20. Beavis, R. C.; Chait, B. T. Rapid, Sensitive Analysis of Protein Mixtures by Mass Spectometry. Proc. Nat. Acad. Sci. U.S.A. 1990, 87, 6873-6877.

21. Bai, J.; Liu, Y. H.; Cain, T. C.; Lubman, D. M. Matrix-Assisted Laser Desorption/Ionization Using an Active Perfluorosulfonated Ionomer Film Substrate. Anal. Chem. 1994, 66, 3423-3430.

22. Wu, K. J.; Steding, A.; Becker, C. H. Matrix-Assisted Laser Desorption Time-of-Flight Mass Spectrometry of Oligonucleotides Using 3-Hydroxypicolinic Acid as an UltravioletSensitive Matrix. Rapid Commun. Mass Spectrom. 1993, 7, 142-146.

23. Bowdler, A. R., Cotter, R. J., Kovtoun, S. V. Combining Mass Correlated Acceleration and a Curved Field Reflectron. Proceedings of the 49th ASMS Conference on Mass Spectrometry and Allied Topics; Chicago, Illinois, May, 2001. 